\title{
Fatores sociodemográficos e comportamentos de risco associados ao consumo do álcool: um recorte do Erica
}

\author{
Socio-demographic factors and risk behaviors associated with alcohol \\ consumption: a cutout of the Erica study
}

Luciana Ramos de Moura', Karine Ferreira dos Santos ${ }^{2}$, Hebert Geraldo de Souza ${ }^{\mathbf{3}}$, Matilde Meire Miranda Cadete $\mathbf{4}^{4}$ Cristiane de Freitas Cunha ${ }^{\mathbf{5}}$

DOI: 10.1590/0103-11042018S411

\footnotetext{
'Faculdade de Ciências Médicas de Minas Gerais - Belo Horizonte (MG) Brasil. Orcid: http://orcid. org/0000-0003-13487041

lulysramos29@yahoo.com.br

2 Universidade Federal de Minas Gerais (UFMG)

- Belo Horizonte (MG),

Brasil.

Orcid: https://orcid. org/0000-0002-33014631

kakaferreira@gmail.com

3 Fundação de Educação para o Trabalho de Minas Gerais (Utramig) - Belo Horizonte (MG), Brasil. Orcid: https://orcid. org/0000-0003-45707301

hebertg.souza@hotmail.com

4 Universidade Federal de Minas Gerais (UFMG)

- Belo Horizonte (MG),

Brasil.

Orcid: http://orcid.

org/0000-0001-8946-

1863

matilde@nescon.medicina.

ufmg.br

5 Universidade Federal de Minas Gerais (UFMG)

- Belo Horizonte (MG),

Brasil.

Orcid: http://orcid.

org/0000-0003-2216-

7904

cristianedefreitascunha@

gmail.com
}

RESUMO O uso de álcool impacta a morbimortalidade na adolescência. Assim, este estudo objetivou identificar a relação entre álcool, variáveis sociodemográficas e comportamentos de risco entre adolescentes do município de Belo Horizonte. Realizaram-se análise descritiva e testes de associação e regressão logística a partir dos dados do Estudo de Riscos Cardiovasculares na Adolescência (Erica). Foi encontrado um consumo de álcool por 22,1\% dos adolescentes. Os resultados da análise multivariada revelaram que não usar o Anticoncepcional Oral (ACO) na última relação aumentou 3,5 vezes as chances de o adolescente fazer uso de bebida alcoólica (OR: 3,5 IC95\% 2,49-4,91). Fumar aumentou 7,25 vezes as chances de o adolescente fazer uso de bebida alcóolica (OR: 7,25 IC95\% 3,7-14,22). Adolescentes do sexo masculino possuem 1,47 vezes mais chances de consumir bebidas alcoólicas que as meninas (OR: 1,47 IC95\% 1,14-1,89). O avançar da idade aumenta 1,36 vezes a chance de o adolescente fazer uso de álcool (OR:1,36 IC95\% 22-1,51). Apresentar maior valor de proxy de riqueza aumentou 1,04 vezes a chance de o adolescente consumir bebida alcoólica (OR: 1,04 IC95\% 1,01-1,07). Assim, conclui-se que as ações em saúde voltadas para a prevenção do consumo do álcool entre adolescentes devem contemplar as diferenças entre as classes sociais, o gênero e a idade, bem como a prevenção do tabagismo e a promoção da saúde sexual e reprodutiva.

PALAVRAS-CHAVE Comportamento do adolescente. Etanol. Assunção de riscos. Anticoncepção.

ABSTRACT Alcohol use impacts morbimortality in adolescence. Thus, this study aimed at identifying the relationship between alcohol, sociodemographic variables and risk behaviors among adolescents from the city of Belo Horizonte. Descriptive analysis and association tests and logistic regression were performed based on the data from the Study of Cardiovascular Risk in Adolescents (Erica). Alcohol consumption was found in $22.1 \%$ of the adolescents. The results of the multivariate analysis revealed that not using Oral Contraceptive (OC) in the last relation increased by 3.5 times the odds of adolescents consuming alcoholic beverage (OR: 3.5 CI95\% 2.49-4.91). Smoking increased the chances of teenagers making use of alcoholic beverage by 7.25 times (OR: 7.25 CI95\% 3.7-14,22). Male adolescents are 1.47 times more likely to consume alcoholic beverages than girls (OR:1.47 CI 95\% 1.14-1.89). Ageing increases 1.36 
times the chance of adolescents using alcohol (OR: 1.36 CI95\% 22-1.51). Presenting higher value of wealth proxy increased 1.04 times the chance of adolescents consuming alcoholic beverage (OR: 1.04 95\% CI 1.01-1.07). Thus, it is concluded that health actions aimed at preventing alcohol consumption among adolescents should contemplate the differences between social classes, gender and age, as well as prevention of smoking and the promotion of sexual and reproductive health.

KEYWORDS Adolescent behavior. Ethanol. Risk-taking. Contraception.

\section{Introdução}

A cada dia, um grande número de adolescentes apresenta condutas que os colocam em risco para as principais causas de morbimortalidade dessa faixa etária'. São os chamados Comportamentos de Risco em Saúde (CRS). Entre eles, estão a inatividade física, o consumo de bebidas alcoólicas, o tabagismo e hábitos alimentares inadequados, afetando um número cada vez mais expressivo de jovens em diferentes países. Entre os CRS, o consumo de álcool merece destaque, uma vez que, anualmente, 1,7 milhão de adolescentes no mundo perde a vida de forma violenta, a maioria pelo uso dessa substância ou associado ao uso de outras drogas ${ }^{2}$. Entre os adolescentes, o álcool é, ainda, um dos responsáveis pelo aumento dos acidentes e óbitos por causas externas ${ }^{3}$.

Apesar dos riscos que apresenta à saúde do adolescente, o álcool é a substância psicotrópica legalizada mais utilizada por adolescentes no Brasil (onde a comercialização é permitida a partir dos 18 anos de idade) $)^{4}$ e no mundo. A iniciação costuma ocorrer precocemente, em idade inferior a 12 anos, com a família e os amigos, em festas, bares e shoppings ${ }^{3}$. Seu consumo causa prejuízos ao desenvolvimento cerebral, favorece a impulsividade e os comportamentos de risco, com repercussões até a vida adulta ${ }^{5}$.

Importante destacar a interferência do álcool em outros comportamentos de risco e agravos à saúde. Autores afirmam que os adolescentes, sob o efeito do álcool, estão mais vulneráveis ao tabagismo, ao uso de drogas ilícitas e ao sexo desprotegido ${ }^{6}$. Estão ainda mais vulneráveis aos acidentes de trânsito, homicídios e suicídios?.

Nas últimas décadas, vários avanços relacionados ao controle do consumo de álcool ocorreram no Brasil. Um exemplo é a proibição da venda de bebidas alcoólicas a menores de 18 anos: vender, fornecer, servir, ministrar ou entregar bebida alcoólica a criança ou adolescente, ainda que gratuitamente, é passível de multa e detenção por dois a quatro anos ${ }^{4}$. Apesar dos avanços, o uso do álcool entre jovens ainda merece atenção, tendo em vista sua prevalência e os agravos associados. Diante do exposto, o presente estudo objetiva avaliar a associação entre o consumo de bebidas alcoólicas e as variáveis sociodemográficas e outros comportamentos de risco para a saúde entre adolescentes da cidade de Belo Horizonte, no estado de Minas Gerais (MG).

\section{Metodologia}

Trata-se de estudo transversal realizado com adolescentes com idade entre 12 e 17 anos, matriculados no sétimo, oitavo e nono anos do ensino fundamental e no primeiro, segundo e terceiro anos do ensino médio nas escolas públicas e privadas de Belo Horizonte. Trata-se de um recorte do Estudo de Riscos 
Cardiovasculares entre Adolescentes (Erica), realizado em 26 capitais do Brasil e no Distrito Federal, cuja coleta de dados se deu entre os anos de 2013 e 2014. O Erica foi realizado em parceria com o Ministério da Saúde do Brasil e intentou estimar a prevalência dos fatores de risco cardiovasculares entre adolescentes, obtendo informações por meio de questionários estruturados, avaliação antropométrica e exames laboratoriais. Todos os escolares das turmas selecionadas foram convidados a participar do estudo. Adolescentes fora da faixa etária elegível, grávidas ou com alguma deficiência física foram excluídos da análise ${ }^{8}$.

A população desta pesquisa correspondeu a uma amostra representativa do município de Belo Horizonte. Foram avaliados 2.547 adolescentes de 12 a 17 anos, matriculados em 43 escolas públicas e particulares da capital mineira. Neste recorte, as variáveis utilizadas foram obtidas por meio do Questionário do Adolescente (QA) e do Questionário da Escola (QE), trabalhados pelo estudo principal. O QA foi instrumento de coleta de dados estruturado, preenchido pelo adolescente e inserido em coletor eletrônico de dados (Personal Digital Assistant - PDA), modelo LG GM750Q. O QA possui 11 blocos temáticos que abordam as condições sociodemográficas e os comportamentos de risco praticados pelo adolescente. $\mathrm{O} Q \mathrm{QE}$, por sua vez, foi preenchido por um pesquisador de campo usando o PDA e investigou a estrutura física da escola, além do preparo e da venda de alimentos nesse ambiente.

Foram considerados em risco aqueles adolescentes que ingeriram bebidas alcoólicas nos últimos 30 dias, em qualquer frequência ou quantidade. Esse critério segue as definições adotadas pela Organização Mundial de Saúde (OMS) e pelo Centro para Prevenção e Controle de Doenças dos Estados Unidos (CDC) 9 .

O consumo de álcool foi associado às variáveis sociodemográficas abordadas pelo Erica, sendo elas: sexo; idade; cor; proxy de riqueza; tipo de escola; oferta e venda de alimentos pela escola.

O proxy de riqueza foi calculado a partir da soma da pontuação atribuída aos bens e itens de conforto considerados a partir da estratificação social do Critério de Classificação Econômica Brasil (CCEB), elaborado pela Associação Brasileira de Empresas de Pesquisa (Abep) ${ }^{10}$. Optou-se pelo uso do proxy de riqueza pela falta de informação em muitos dos itens necessários para a classificação econômica segundo a Abep, já que era possível aos adolescentes, ao responder ao questionário do aluno, selecionar em pelo menos um dos itens essenciais à classificação: 'não sei/não me lembro/prefiro não responder'. A exclusão desses sujeitos representaria uma perda importante e heterogeneamente distribuída da amostra, comprometendo a qualidade da investigação.

Foram estudadas, também, as associações entre o uso de álcool nos últimos 30 dias (variável desfecho) e os comportamentos de risco para a saúde investigados pelo Erica, que coincidem com aqueles monitorados pelo $\mathrm{CDC}^{9}$, sendo eles:

a) Comportamentos sexuais que contribuem para gravidez involuntária e infecções sexualmente transmissíveis - não uso da camisinha; não uso do Anticoncepcional Oral (ACO) (ambos em referência à última relação) ${ }^{\mathbf{n 1}}$;

b) Atividade física inadequada - realização de menos de 300 minutos de atividade física por semana ${ }^{\mathbf{1 2}}$;

c) Comportamento alimentar não saudável - não tomar café da manhã (todos os dias ou quase todos os dias); almoçar e jantar na frente da TV (todos os dias ou quase todos os dias); comer petiscos na frente da TV, do computador ou do videogame (quase todos os dias ou todos os dias); e não beber água (ou beber menos de dois copos por dia) ${ }^{\mathbf{1 3}}$;

d) Uso do tabaco - se houve consumo nos últimos 30 dias?.

Todas as análises realizadas levaram em consideração a amostragem aleatória complexa com pesos amostrais. Foram apresentadas 
as medidas descritivas mínimo, máximo, mediana, média, Desvio-Padrão (DP) e Intervalo de Confiança (IC) de 95\%, além de percentuais para descrever os resultados das variáveis estudadas. A associação entre duas variáveis categóricas de interesse foi realizada utilizando o teste do Qui-quadrado.

Com o objetivo de comparar os dois grupos independentes quanto à medida de uma variável do tipo escalar, foi utilizado o teste $t$ de Student. O modelo de regressão logística foi utilizado na identificação de variável(eis)/ fator(es) associados, de forma conjunta, na ocorrência de determinado evento de interesse. O teste que avalia a bondade do ajuste do modelo logístico foi utilizado com o objetivo de avaliar se as variáveis utilizadas para explicar determinado desfecho são ou não suficientes. As variáveis categóricas foram dicotomizadas, isto é, foram transformadas em variáveis do tipo Dummy e utilizadas para explicar o efeito que diferentes níveis de uma variável não métrica (variável categórica) têm na previsão da variável dependente/desfecho (com comportamento de risco). A magnitude das associações foi determinada pela Odds Ratio (OR), com Intervalo de Confiança de 95\% (IC 95\%). Todos os resultados foram considerados significativos para uma probabilidade de significância inferior a 5\% $(p<0,05)$, tendo, portanto, pelo menos $95 \%$ de confiança nas conclusões apresentadas. Foi utilizado o software estatístico Stata/SE 13.0 for Windows na análise dos dados.

O presente estudo foi aprovado pelos Comitês de Ética da Universidade Federal do Rio de Janeiro (Processo 45/2008) e, em Minas Gerais, o estudo foi aprovado sob o parecer $\mathrm{n}^{\mathrm{o}}$ ETIC 224/2009. A privacidade dos alunos e a confidencialidade das informações foram garantidas em todas as etapas da pesquisa.

\section{Resultados}

Na caracterização da população estudada, constatou-se que, entre os adolescentes escolares de Belo Horizonte, a estrutura familiar é predominantemente biparental (56,9\%). Entretanto, cerca de um terço deles $(34,6 \%)$ reside em lares com a presença apenas da mãe. Sobre a escolaridade da mãe, $27,6 \%$ dos adolescentes não souberam, não lembraram ou preferiram não responder. O proxy de riqueza evidenciou pontuação média de 22,9 para os bens e itens de conforto (tabela 1). Conforme dados apresentados na tabela 1, o consumo de bebidas alcoólicas entre adolescentes, no último mês, foi de 22,1\% (IC95\% 19,5-24,8).

Tabela 1. Caracterização sociodemográfica e consumo de álcool entre adolescentes no município de Belo Horizonte, MG, 2017

\begin{tabular}{lr}
\hline Características & $\%$ \\
\hline Sexo & 50,0 \\
\hline Feminino & 50,0 \\
Masculino & 17,0 \\
\hline Idade & 17,4 \\
\hline 12 anos & 16,8 \\
13 anos & 17,6 \\
14 anos & 16,7 \\
15 anos & 14,5 \\
17 anos & \\
\hline
\end{tabular}


Tabela 1. (cont)

\begin{tabular}{|c|c|}
\hline Cor & \\
\hline Branca & 33,7 \\
\hline Parda & 54,3 \\
\hline Preta & 8,4 \\
\hline Outras & 3,6 \\
\hline \multicolumn{2}{|l|}{ Estrutura familiar } \\
\hline Mora com pai e mãe & 56,9 \\
\hline Mora só com a mãe & 34,6 \\
\hline Mora só com o pai & 4,0 \\
\hline Não mora com pai e mãe & 4,5 \\
\hline \multicolumn{2}{|l|}{ Escolaridade da mãe } \\
\hline Analfabeta/Menos de 1 ano de instrução & 1,2 \\
\hline 1 a 3 anos do ensino fundamental & 4,7 \\
\hline 4 a 7 anos do ensino fundamental & 11,9 \\
\hline Ensino fundamental completo & 6,7 \\
\hline Ensino médio incompleto & 6,8 \\
\hline Ensino médio completo & 19,8 \\
\hline Superior incompleto & 3,5 \\
\hline Superior completo & 17,8 \\
\hline Não sei/não lembro/prefiro não responder & 27,6 \\
\hline Proxy de riqueza & $22,9 \pm 0,4(10$ a 38) \\
\hline \multirow[t]{2}{*}{ Consumo de bebidas alcoólicas } & Prevalência (IC 95\%) \\
\hline & $22,1(19,5 ; 24,8)$ \\
\hline
\end{tabular}

Fonte: Dados da pesquisa.

A tabela 2 demonstra a associação entre os comportamentos de risco e os fatores sociodemográficos relacionados ao uso de bebida alcoólica por meio de análises univariadas e multivariadas. Entre os adolescentes que não usaram ACO na última relação sexual, 47,1\% consumiram bebidas alcoólicas. A análise multivariada evidenciou que não usar o ACO na última relação sexual aumentou 3,5 vezes as chances de o adolescente fazer uso de bebida alcoólica (OR: 3,5 IC95\% 2,49-4,91). 
Tabela 2. Avaliação da influência dos comportamentos de risco e fatores sociodemográficos no uso de bebida alcoólica entre adolescentes da cidade de Belo Horizonte (MG), 2017

\begin{tabular}{|c|c|c|c|c|c|}
\hline \multirow[t]{3}{*}{ Variável } & \multicolumn{3}{|c|}{ Análise univariada } & \multirow{2}{*}{\multicolumn{2}{|c|}{$\begin{array}{c}\text { Análise multivariada } \\
\text { Modelo final }\end{array}$}} \\
\hline & \multicolumn{2}{|c|}{ Uso de bebida alcoólica } & \multirow[t]{2}{*}{ Teste estatístico } & & \\
\hline & Sim & Não & & $\mathrm{p}$ & OR (IC 95\%) \\
\hline \multicolumn{6}{|c|}{ Comportamentos de risco (ref. Sim) } \\
\hline \multicolumn{6}{|c|}{ Comportamento sexual - camisinha } \\
\hline Sim & 21,2 & 78,8 & $\chi^{2}=55,5 / p<0,001$ & & \\
\hline Não & 45,8 & 54,2 & & & \\
\hline \multicolumn{6}{|c|}{ Comportamento sexual - anticoncepcional (ref. Sim) } \\
\hline $\operatorname{sim}$ & 16,8 & 83,2 & $\chi 2=206,6 / p<0,001$ & $\beta=1,25 / p<0,001$ & $3,5(2,49 ; 4,91)$ \\
\hline Não & 47,1 & 52,9 & & & \\
\hline \multicolumn{6}{|c|}{ Uso de tabaco (ref. Não) } \\
\hline Não & 20,5 & 79,5 & $\chi 2=174,1 / p<0,001$ & $\beta=1,98 / p<0,001$ & $7,25(3,7 ; 14,22)$ \\
\hline $\operatorname{sim}$ & 78,1 & 21,9 & & & \\
\hline \multicolumn{6}{|c|}{ Prática de atividades físicas inadequadas (ref. Não) } \\
\hline Não & 22,3 & 77,7 & $\chi 2=0,46 / p=0,622$ & & \\
\hline Sim & 23,5 & 76,5 & & & \\
\hline \multicolumn{6}{|c|}{ Hábitos alimentares inadequados (ref. Não) } \\
\hline Não & 17,4 & 82,6 & $\chi 2=3,75 / p=0,097$ & & \\
\hline Sim & 23,4 & 76,6 & & & \\
\hline \multicolumn{6}{|c|}{ Sexo (ref. Feminino) } \\
\hline Feminino & 24,9 & 75,1 & $\chi^{2}=5,40 / p=0,037$ & $\beta=-0,38 / p=0,003$ & $0,68(0,53 ; 0,88)$ \\
\hline Masculino & 21,0 & 79,0 & & & \\
\hline
\end{tabular}

Associação significativa também foi observada entre o consumo de bebida alcoólica e o tabaco: entre os adolescentes que fumaram nos últimos 30 dias, 78,1\% também fizeram uso de bebida alcoólica. Fumar aumentou 7,25 vezes as chances de 0 adolescente fazer uso de bebida na presente amostra (OR: 7,25 IC95\% 3,7-14,22).

A análise multivariada não evidenciou associações significativas entre o consumo de bebidas alcoólicas e os demais comportamentos de risco dos investigados.

No que diz respeito aos fatores sociodemográficos, os resultados da análise multivariada revelaram associação entre consumo de bebida alcoólica e gênero, idade, proxy de riqueza do adolescente.

Quando comparadas aos meninos, as meninas consumiram maior quantidade de bebida alcoólica (21,0\% de uso entre meninos e $24,9 \%$ de uso entre meninas, $p=0,037$ ). Apesar disso, os resultados da análise multivariada apuraram que, em Belo Horizonte, os adolescentes do sexo masculino possuem 1,47 (1/OR) vez mais chances de consumir bebidas alcoólicas (OR: 1,47 IC95\% $1,14-1,89)$ que as meninas.

O consumo de bebidas alcoólicas também aumentou de forma significativa com a idade: aos 12 anos de vida, 6,5\% dos adolescentes relataram uso de bebidas, ao passo que, aos 17 anos, o consumo já atinge $36,9 \%$ da amostra $(\mathrm{p}<0,001)$. A análise multivariada acusou que $o$ avançar da idade aumenta 1,36 vezes a chance de o adolescente fazer uso de álcool.

O proxy de riqueza foi maior entre os 
adolescentes que relataram consumo de bebida alcoólica no último mês (23,6 pontos). Apresentar maior valor de proxy de riqueza aumentou 1,04 vezes a chance de 0 adolescente consumir bebida alcoólica (OR: 1,04 IC95\% 1,01-1,07).
O maior percentual de adolescentes que beberam nos últimos 30 dias foi observado entre os que não residem com o pai e a mãe (35,8\%). Já o menor consumo do álcool ocorreu entre os que residem com o pai e a mãe $(20,6 \%)$ $(\mathrm{p}=0,017)$.

Tabela 3. Avaliação da influência dos comportamentos de risco e fatores sociodemográficos no uso de bebida alcoólica entre adolescentes da cidade de Belo Horizonte (MG), 2017

\begin{tabular}{|c|c|c|c|c|c|}
\hline \multirow[t]{3}{*}{ Variável } & \multicolumn{3}{|c|}{ Análise univariada } & \multirow{2}{*}{\multicolumn{2}{|c|}{$\begin{array}{c}\text { Análise multivariada } \\
\text { Modelo final }\end{array}$}} \\
\hline & \multicolumn{2}{|c|}{ Uso de bebida alcoólica } & \multirow[t]{2}{*}{ Teste estatístico } & & \\
\hline & Sim & Não & & $\mathrm{p}$ & OR (IC 95\%) \\
\hline \multicolumn{6}{|l|}{ Idade } \\
\hline 12 anos & 6,5 & 93,5 & $\chi 2=160,4 / p<0,001$ & $\beta=0,3 / p<0,001$ & $1,36(1,22 ; 1,51)$ \\
\hline 13 anos & 15,3 & 84,7 & & & \\
\hline 14 anos & 18,1 & 81,9 & & & \\
\hline 15 anos & 29,3 & 70,7 & & & \\
\hline 16 anos & 33,8 & 66,2 & & & \\
\hline 17 anos & 36,9 & 63,1 & & & \\
\hline \multicolumn{6}{|c|}{ Estrutura familiar (ref. Mora com mãe e pai) } \\
\hline Mora com mãe e pai & 20,6 & 79,4 & $\chi 2=16,80 / p=0,017$ & & \\
\hline Mora somente com mãe & 24,8 & 75,2 & & & \\
\hline Mora somente com pai & 26,9 & 73,1 & & & \\
\hline Não mora com mãe e pai & 35,8 & 64,2 & & & \\
\hline \multicolumn{6}{|l|}{ Cor (ref. Branca) } \\
\hline Branca & 24,5 & 75,5 & $\chi 2=5,54 / p=0,337$ & & \\
\hline Parda & 21,9 & 78,1 & & & \\
\hline Preta & 27,6 & 72,4 & & & \\
\hline Outras & 18,1 & 81,9 & & & \\
\hline Proxy de riqueza & $\begin{array}{r}23,6(22,5 ; 24,8) \\
M d=24,0\end{array}$ & $\begin{array}{r}22,7(21,9 ; 23,5) \\
M d=23,0\end{array}$ & $t=1,85 / p=0,071$ & $\begin{array}{r}\beta=0,04 / p= \\
0,024\end{array}$ & $\begin{array}{r}1,04(1,01 ; \\
1,07)\end{array}$ \\
\hline \multicolumn{6}{|l|}{ Tipo de escola (ref. Privada) } \\
\hline Privada & 25,5 & 74,5 & $\chi 2=2,75 / p=0,596$ & & \\
\hline Pública & 22,2 & 77,8 & & & \\
\hline
\end{tabular}

Notas: Base de dados: análise univariada: 2.547 adolescentes; análise multivariada: 2.159 adolescentes.

Medida de bondade de ajuste do modelo ( $p=0,128)$; análise univariada: teste Qui-quadrado $(\chi 2)$ ou teste t de Student ( $t$ ) / análise multivariada: regressão logística. Os valores apresentados na tabela para a variável proxy de riqueza referem-se a / Md (mediana), e, para as demais variáveis, são percentuais. 


\section{Discussão}

Ao analisar o consumo de álcool por adolescentes belo-horizontinos e os fatores associados a isso, este estudo constatou que, aproximadamente, um quarto deles fez uso de bebida alcoólica em pelo menos uma ocasião nos 30 dias anteriores à pesquisa. Tal constatação pode ser considerada grave, visto que, sob efeito do álcool, aumentam as chances de envolvimento em situações de risco ${ }^{6,7}$. A amostra para a capital mineira da Pesquisa Nacional de Saúde do Escolar (PeNSE), realizada em 2009, encontrou resultados ainda mais alarmantes: na ocasião, aproximadamente $30,5 \%$ de adolescentes haviam consumido álcool pelo menos uma vez nos últimos 30 dias. Apesar da redução, o consumo de álcool em Belo Horizonte continua elevado na comparação com outras capitais brasileiras, como Natal e Macapá, que encontraram frequências de 11,8 e $13,1 \%$, respectivamente ${ }^{14}$.

O Erica, realizado entre os anos de 2013 e 2014 , notificou $21 \%$ de consumo médio de bebidas alcoólicas no País, resultado próximo do encontrado na capital mineira (22,1\%). O consumo de álcool foi mais acentuado na região Sul (27,5\%) e menos acentuado na região Norte $(14,8 \%)^{5}$.

Em Belo Horizonte, o avanço da idade favoreceu o consumo de bebida alcoólica, resultado semelhante ao encontrado por outros autores ${ }^{5}$. Cabe ressaltar que o presente estudo foi realizado com adolescentes de 12 a 17 anos, que, de acordo com a legislação nacional em vigor, não deveriam ter acesso à compra de bebidas alcoólicas. No entanto, por ser socialmente aceito, o consumo de álcool pode sofrer banalização por parte da família, dos amigos e da sociedade, contribuindo, assim, para o acesso e o consumo dessa substância entre adolescentes ${ }^{15}$.

Ao se controlar o efeito das outras variáveis sociodemográficas e comportamentais abordadas neste estudo, os meninos apresentaram mais vulnerabilidade para o consumo de álcool quando comparados às meninas. Esses resultados diferem dos da PeNSE 2012, que evidenciou um maior consumo do álcool pelo sexo feminino ${ }^{6}$. Importante ressaltar que, no presente estudo, o consumo de bebidas alcoólicas também foi frequente entre moças, o que pode apontar novas tendências de identidades de gênero que merecem monitoramento.

Estudos sugerem que residir com ambos os pais promove efeito protetor ao hábito de beber entre jovens ${ }^{16}$, associação não observada no presente estudo. Esse resultado sugere que exista um distanciamento entre os adolescentes e suas respectivas famílias. No entanto, há necessidade de novas investigações para que essa questão seja melhor compreendida.

Sabe-se que a participação ativa da família nos momentos de transformação, entre eles, a adolescência, ajuda a minimizar possíveis condutas de risco. Pesquisas apontam que filhos cujos pais estão mais atentos às suas atividades envolvem-se menos com álcool, outras drogas e tabaco ${ }^{\mathbf{1 6}}$. Famílias que contam com a presença da figura materna e paterna oferecem mais proteção contra o uso e a dependência de drogas. A convivência e a coesão familiares, assim como a participação conjunta de atividades com a família exercem efeito protetor na prevenção contra o uso de álcool e outras drogas ${ }^{17}$.

O consumo de bebida alcoólica foi mais elevado entre os adolescentes que apresentavam maior valor para o proxy de riqueza. $\mathrm{Da}$ mesma forma, há registros na literatura que relacionam o consumo de álcool às classes sociais mais elevadas ${ }^{18}$. Investigando estudantes universitários do município de São Paulo, identificou-se que alunos com renda familiar alta têm elevado risco de consumo de drogas ${ }^{19}$. Entretanto, estudos mostram, também, consumo expressivo de álcool na adolescência e vida adulta por indivíduos com menor poder aquisitivo ${ }^{\mathbf{2 0}}$. Os achados sugerem que um maior poder aquisitivo pode favorecer o acesso a bebidas em Belo Horizonte, facilitado pelo próprio ambiente familiar e social que o adolescente frequenta.

As alterações dos sentidos e a menor percepção do risco provocadas pelo álcool aumentam 
a vulnerabilidade do sujeito a outros CRS, a exemplo dos acidentes, suicídios, violência, gravidez não planejada e transmissão de doenças por via sexual ${ }^{21}$. Esses dados podem explicar a associação entre o uso de bebida alcoólica e não uso do ACO, encontrada no presente estudo e já descrita na literatura ${ }^{22}$. Por se tratar de uma droga lícita e de fácil acesso, o álcool é um dos principais antagonistas da vivência saudável da sexualidade, contribuindo para a vulnerabilidade relacionada a gravidez não planejada e Infecções Sexualmente Transmissíveis (ISTs) ${ }^{\mathbf{2 2}}$. De acordo com pesquisa desenvolvida em 1999 pelo Harvard Campus Alcohol Study, jovens que bebem pela primeira vez antes dos 13 anos de idade têm o dobro de chances de praticar sexo não planejado e 2,2 vezes mais chances de se envolverem em relação sexual desprotegida, quando comparados com estudantes que iniciam o uso de bebida alcoólica após os $19 \operatorname{anos}^{23}$.

A literatura identifica que o uso de substâncias psicoativas costuma produzir efeito multiplicador, em que o consumo de uma substância aumenta as chances de uso das outras ${ }^{24}$, o que pode explicar a relação entre o consumo de álcool e tabaco encontrada neste estudo. Uma hipótese para tal fenômeno é a de que o álcool e as demais drogas suprem, de forma ilusória, os desejos do adolescente, causando a ideia de invulnerabilidade e aumentando, assim, as chances de envolvimento em situações de risco 22 .

\section{Conclusões}

As variáveis sociodemográficas e os comportamentos de risco para a saúde impactam o consumo de álcool entre adolescentes. Atenção especial merece ser direcionada para os adolescentes com idades mais avançadas, do sexo masculino e com maior proxy de riqueza. Adolescentes sexualmente ativos que não fazem uso do ACO e adolescentes tabagistas também estiveram entre os mais vulneráveis.
Desse modo, a prevenção ao consumo de álcool deve ser realizada em conjunto com abordagens voltadas para a prevenção do tabagismo e a prática de sexo seguro.

Assim, mais que a proposição de estratégias prescritivas, é necessário o desenvolvimento de mecanismos de proteção, prevenção e acolhimento que deem suporte ao adolescente por meio de intervenções que estimulem escolhas de vida saudáveis e de qualidade, desmitificando a falsa ideia de autonomia propiciada por substâncias psicoativas. Contribuir para que a família e a escola sejam ambientes de apoio, escuta e acompanhamento dos adolescentes potencializará as ações, bem como o envolvimento dos profissionais da saúde, dos meios de comu-nicação e da sociedade em geral. A identificação dos grupos mais expostos, a partir da valorização das diferenças de sexo, idade e classe social, poderá viabilizar ações que sejam mais bem-sucedidas por contemplarem os adolescentes em sua diversidade.

O presente estudo não investigou algumas variáveis importantes, entre elas, o uso de drogas ilícitas e a influência dos amigos no consumo de álcool e tabaco. Tais variáveis não compuseram o instrumento de coleta de dados do estudo principal, o que impossibilitou a análise no presente recorte. Ressalta-se, de todo modo, que a representatividade amostral para a cidade de Belo Horizonte, bem como associações encontradas, conferem originalidade e relevância para esta investigação.

\section{Colaboradores}

Moura LR contribuiu para a concepção, planejamento, análise e interpretação dos dados, elaboração do rascunho, revisão crítica do conteúdo e aprovação final do manuscrito. Santos KF e Souza HG contribuíram para a revisão crítica do conteúdo. Cadete MMM e Cunha CF contribuíram para a concepção, planejamento, análise e interpretação dos dados. 


\section{Referências}

1. Kann L, McManus T, Harris WA, et al. Youth risk behavior surveillance - United States, 2015. MMWR Surveill. Summ. 2016; 65(6):1-174.

2. Farias Junior JC. Associação entre prevalência de inatividade física e indicadores de condição socioeconômica em adolescentes. Rev. Bras. Med. Esporte. 2008; 14:109-14.

3. Elicker E, Palazzo LS, Aerts DRGC, et al. Uso de álcool, tabaco e outras drogas por adolescentes escolares de Porto Velho-RO, Brasil. Epidemiol. Serv. Saúde. 2015; 24(3):399-410.

4. Brasil. Lei $\mathrm{n}^{0} 13.106$, de 17 de março de 2015. Altera a lei $\mathrm{n}^{\circ}$ 8.069, de 13 de julho de 1990 - Estatuto da Criança e do Adolescente, para tornar crime vender, fornecer, servir, ministrar ou entregar bebida alcoólica a criança ou a adolescente; e revoga o inciso I do Art. 63 do decreto-lei no 3.688 , de 3 de outubro de 1941 - Lei das Contravenções Penais. Diário Oficial da União. 13 Out 2015 [acesso em 2015 nov 12]. Disponível em: http://www.planalto.gov.br/ccivil_03/_ ato2015-2018/2015/lei/ 113106.htm.

5. Coutinho ESF, França-Santos D, Magliano ES, et al. ERICA: padrões de consumo de bebidas alcoólicas em adolescentes brasileiros. Rev. Saúde Pública. 2016; 50(supl1):8s.

6. Malta DC, Oliveira-Campos M, Prado RR, et al. Uso de substâncias psicoativas, contexto familiar e saúde mental em adolescentes brasileiros, Pesquisa Nacional de Saúde dos Escolares (PeNSE 2012). Rev. Bras. Epidemiol. [internet]. 2014 [acesso em 2017 maio 31]; 17(supl1):46-61. Disponível em: https://repositorio.observatoriodocuidado.org/bitstream/handle/719/1/rbe.S1415-790X2014000500046.pdf.

7. Garnica Wesselovicz AA, Sousa TG, Nobuyoshi Kaneshima E, et al. Fatores associados ao consumo de bebidas alcoólicas pelos adolescentes de uma Escola Pública da cidade de Maringá, Estado do Paraná. Acta Sci., Health Sci. 2008; 30(2):161-166.
8. Vasconcellos MTL, Silva PLN, Szklo M, et al. Diseño de la muestra del estudio de riesgos cardiovasculares en adolescentes (ERICA). Cad. Saúde Pública. 2015;31(5):921-930.

9. Centers for Disease Control and Prevention (United States). Youth risk behavior surveillance: United States, 2005. MMWR Surv. Summ. 2006; 55(ss-5):1-108.

10. Associação Brasileira de Empresas de Pesquisa [internet]. [São Paulo]: Critério Brasil de classificação econômica; 2012 [acesso em 2017 maio 31]. Disponível em: http://www.abep.org/criterio-brasil.

11. Borges ALV, Fujimori E, Kuschnir MCC, et al. ERICA: início da vida sexual e contracepção em adolescentes brasileiros. Rev. Saúde Pública [internet]. 2016 [acesso em 2017 jun 4]; 50(supl1):15s. Disponível em: http://www.scielo.br/pdf/rsp/v50s1/pt_0034-8910-rsp-S01518-87872016050006686.pdf.

12. Farias Júnior JC, Lopes AS, Mota J, et al. Prática de atividade física e fatores associados em adolescentes no nordeste do Brasil. Rev. Saúde Pública [internet]. 2012 [acesso em 2017 jun 6]; 46(3):505-515. Disponível em: https://www.scielosp.org/article/rsp/2012. v46n3/505-515/pt/.

13. Barufaldi LA, Abreu GZ, Oliveira JS, et al. ERICA: prevalência de comportamentos alimentares saudáveis em adolescentes brasileiros. Rev Saúde Publica. 2016; 50(supl1):6s.

14. Prefeitura Municipal de Belo Horizonte, Secretaria Municipal de Saúde [internet]. Belo Horizonte: Prevalência dos fatores de risco e de proteção comportamentais em adolescentes de Belo Horizonte Pesquisa nacional de saúde do escolar (PeNSE); 2012 [acesso em 2017 jun 6]. Disponível em: https://prefeitura.pbh.gov.br/sites/default/files/estrutura-de-governo/saude/2018/publicacaoes-da-vigilancia-em-saude/boletim_PeNSE.pdf.

15. Lopes AP, Ganassin GS, Marcon SS, et al. Abuso de bebida alcoólica e sua relação no contexto familiar. 
Estud. Psicol. (Natal). 2015; 20(1):22-30.

16. Paiva FS, Rozani TM. Estilos parentais e consumo de drogas entre adolescentes: revisão sistemática. Psicol. Estud. 2009; 14(1):117-83.

17. Guimarães AB, Hochgraf PB, Brasiliano S, et al. Aspectos familiares de meninas adolescentes dependentes de álcool e drogas. Rev. de Psiquiat. Clín. 2009; 36(2):69-74.

18. Almeida-Filho N, Lessa I, Magalhães L, et al. Alcohol drinking patterns by gender, ethnicity, and social class in Bahia, Brazil. Rev. Saúde Pública. 2004; $38: 45-54$.

19. Silva LV, Malbergier A, Stempliuk VD, et al. Fatores associados ao consumo de álcool e drogas entre estudantes universitários. Rev. Saúde Pública. 2006; 40:280-8.

20. Mendoza Sassi RA, Béria JU. Prevalence of alcohol use disorders and associated factors: a population based study using AUDIT in southern Brazil. Addiction. 2003 jun 1; 98(6):799-804.
21. Reboussin BA, Song EY, Shrestha A, et al. A latent class analysis of underage problem drinking: Evidence from a community sample of 16-20 year olds. Drug Alcohol Depend. 2006; 83(3):199-209.

22. Sampaio Filho FJ, Sousa PR, Vieira NF, et al. Percepção de risco de adolescentes escolares na relação consumo de álcool e comportamento sexual. Rev. Gaúcha Enferm. 2010; 31(3):508.

23. Hingson R, Heeren T, Winter MR, et al. Early age of first drunkenness as a factor in college students' unplanned and unprotected sex attributable to drinking. Pediatrics. 2003; 111(1):34-41.

24. Lisha NE, Sussman S. Relationship of high school and college sports participation with alcohol, tobacco, and illicit drug use: A review. Addic. behav. 2010; 35(5):399-407.

Recebido em 03/06/2018

Aprovado em 03/10/2018

Conflito de interesses: inexistente

Suporte financeiro: não houve 